\title{
Rapid consolidation of powdered materials by induction hot pressing
}

\author{
Aaron D. LaLonde, Teruyuki Ikeda, and G. Jeffrey Snyder \\ Materials Science, California Institute of Technology, Pasadena, \\ California 91125, USA
}

(Received 15 November 2010; accepted 5 December 2010; published online 15 February 2011)

\begin{abstract}
A rapid hot press system in which the heat is supplied by RF induction to rapidly consolidate thermoelectric materials is described. Use of RF induction heating enables rapid heating and consolidation of powdered materials over a wide temperature range. Such rapid consolidation in nanomaterials is typically performed by spark plasma sintering (SPS) which can be much more expensive. Details of the system design, instrumentation, and performance using a thermoelectric material as an example are reported. The Seebeck coefficient, electrical resistivity, and thermal diffusivity of thermoelectric $\mathrm{PbTe}$ material pressed at an optimized temperature and time in this system are shown to agree with material consolidated under typical consolidation parameters. (C) 2011 American Institute of Physics. [doi:10.1063/1.3534080]
\end{abstract}

\section{INTRODUCTION}

The use of spark plasma sintering (SPS), and many variants has grown rapidly in recent years. ${ }^{1}$ While it is not clear that sparking or plasma is involved, ${ }^{2}$ SPS systems have become widely used for the consolidation of fine grained and nanomaterials because of its speed compared to conventional (pressureless) sintering or even conventional hot pressing. ${ }^{3}$ Such rapid pressing is particularly important when consolidating nanomaterials in order to limit grain growth. ${ }^{4,5}$ Much of the advantage of SPS may be due to the rapid heating rate during uniaxial pressing, a parameter that could be achieved by other means that avoid the high currents and other effects of SPS.

The hot press system described here was developed to rapidly consolidate thermoelectric materials over a large temperature range (400-2300 K) within an inert atmosphere and is referred to as rapid hot pressing (RHP). The heat in this system is provided by an induction coil operated in the RF range applied directly to a graphite die acting as a susceptor. Utilizing the hydraulic control system during processing, the consolidation of samples can be monitored to determine the minimum required pressing time.

Processing of many materials ultimately requires consolidation of powdered material into dense ingots or discs for subsequent steps in device fabrication, thermoelectric materials are one example. The most prevalent consolidation techniques applied to thermoelectric materials are hot pressing (HP) using resistance heaters and SPS. While each method is capable of consolidating material, there are disadvantages associated with each making an alternative approach to consolidation desirable.

There are many disadvantages of the HP and SPS systems that are not present in the RHP method making it an attractive alternative for producing consolidated thermoelectric materials. Table I highlights six important aspects of each consolidation method compared relative to each other. In regards to the initial cost of the system fabrication, as well as maintenance costs, the RHP is the least expensive system as it is constructed from readily available and affordable components.
The overall footprint of the RHP system is reduced mainly due to the compact size of the induction power supply, which is drastically smaller than the necessary power supply hardware for either a HP or SPS system. The heating rate of a HP system is relatively slow as the heat is radiated throughout the whole chamber. In an SPS system the pressing rods and die body are resistively heated achieving a heating rate comparable to RHP; however, during RHP only the die body is heated inside the induction coil enabling faster cooling of the die and chamber. The more directed heating and faster cooling of RHP compared to SPS should also enable higher peak temperature in a RHP as induction heating is known to achieve temperatures above $2773 \mathrm{~K}$ at the susceptor. ${ }^{6} \mathrm{~A}$ significant and poorly understood characteristic of the SPS method is the electrically driven transport of ions within the material during consolidation leading to significant chemistry variation in the consolidated material, ${ }^{7}$ an affect that neither the HP or the RHP is plagued with. From a design flexibility standpoint the ability to use different size pressing dies without making other changes to the system adds versatility to the system. In both a HP and RHP system various die sizes can be used with the same heating element or induction coil without additional changes to the system; however, changing die sizes in an SPS system will alter the heating characteristic of the die as it is a function of the die resistance and the current flow through the die. Additionally, the heating elements and related connections in a HP system are expensive custom machined pieces that are very fragile. Similarly in an SPS system, the electrodes are large precision machined pieces and considerable cost and effort is made to minimize contact resistances between connections. ${ }^{8}$ It has also been shown in SPS systems that the temperature distribution within the pressing die is dependent on the material being consolidated and the electrode design and configuration. ${ }^{2}$ In the RHP system the induction coil is easily made in our laboratory from readily available inexpensive copper tubing and can be replace or redesigned immediately. Lastly, the ease of scale up for each technique is important for consolidation of large quantities of material for production purposes. Consolidation of larger amounts of material invariably means using larger 
TABLE I. Comparison of important aspects of consolidation techniques.

\begin{tabular}{lccc}
\hline \hline System & HP & SPS & RHP \\
\hline Cost & Med & High & Low \\
System size & Med & Large & Small \\
Heating rate & Slow & Fast & Fast \\
Chemistry effect & None & Problem & None \\
Design flexibility & Med & Small & Large \\
Scale up & Easy & Hard & Easy \\
\hline \hline
\end{tabular}

diameter dies, chambers, power supplies, and in the case of HP and RHP a larger heating element or induction coil. Scale up of SPS is relatively difficult compared to HP and RHP because of the size and cost of the necessary power supply.

In this article a custom-built induction hot press capable of rapidly consolidating material over a wide temperature range under selectable atmospheres is described. Densification of samples takes place under uniaxial pressure in a graphite die acting as a susceptor within a RF induction coil. Thermoelectric material produced in our laboratory has been consolidated in this system and the Seebeck coefficient, resistivity, and thermal diffusivity have been measured and are reported to demonstrate that this method is capable of producing functional material quickly at low and high temperatures.

\section{EXPERIMENTAL SETUP}

The schematic for the experimental setup is shown in Fig. 1. The system was retrofitted onto an Instron 1350

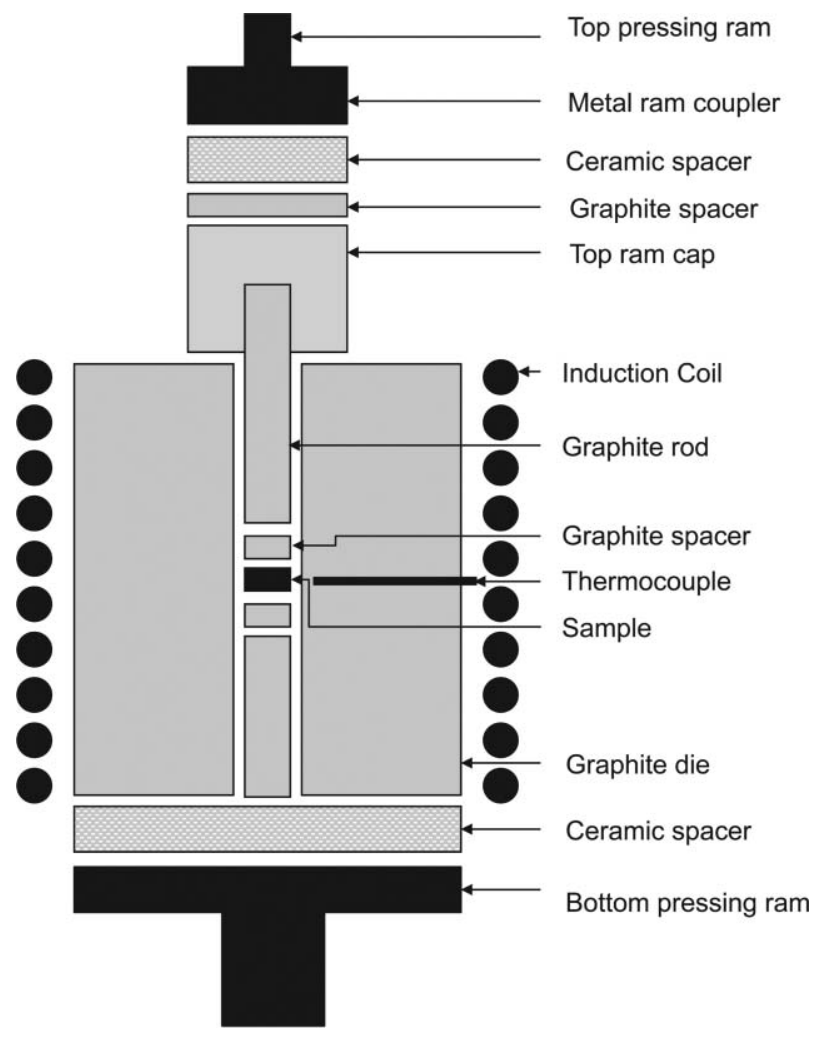

FIG. 1. Schematic of induction hot press setup.

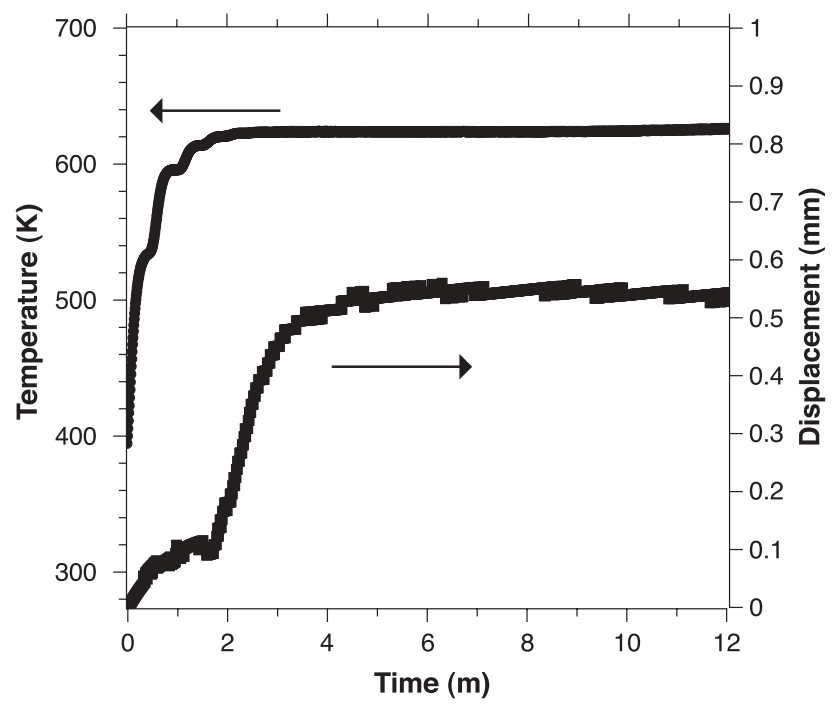

FIG. 2. Temperature vs ram displacement for consolidation of sample at 623 $\mathrm{K}$ for $10 \mathrm{~min}$.

mechanical testing load frame and hydraulic system allowing for precise control of the pressing ram position and applied load. A Centorr M60 multipurpose vacuum chamber was incorporated into the load frame with vacuum bellows attached to the pressing rams. The chamber walls and additional copper cooling plates are cooled with a closed-loop water chiller that additionally serves to cool the diffusion pump, hydraulic system, induction coil, and induction power supply. The system can be operated under vacuum or backfilled with a desired gas. The typical vacuum level before inert gas backfilling is $3 \times 10^{-5}$ Torr achieved in $1 \mathrm{~h}$, while the ultimate pressure of the system is approximately $3 \times 10^{-6}$ Torr. The chamber has feedthroughs for up to four thermocouples, one of which is used for control of the die temperature. The other thermocouples can be used to monitor the temperature of the chamber, steel pressing ram, and/or additional locations within the die.

The process control thermocouple is located $3 \mathrm{~mm}$ from the sample in the die and is monitored using a programmable digital controller that supplies input to the induction power supply system creating a feedback loop that controls the temperature of the die. Although the thermocouple passes through the induction coil, the accuracy of this thermocouple configuration has been verified by inserting an additional thermocouple in the top of the die extending to the sample location. The penetration depth, the thickness of the layer around the outside of the graphite die where the majority (87\%) of the heat is developed by the current, is approximately $8 \mathrm{~mm}$. The graphite die acts as a susceptor as it converts the electromagnetic energy to heat and conducts the heat through the die to the sample. We have successfully tested this system to $1450 \mathrm{~K}$ using $20 \mathrm{~kW}$ from the induction heater and even higher temperatures are possible with this $25 \mathrm{~kW}$ power supply. The die that is used in the system is $76 \mathrm{~mm}$ in diameter with a $12 \mathrm{~mm}$ bore through the center of the die body and can be heated at a rate of $620 \mathrm{~K} / \mathrm{min}$. Different sized die bodies can be used within the same induction coil and the bore diameter in the die can be varied to allow different sample sizes. 
When loading the die for pressing the first piece placed in the die is a piece of graphite rod, this piece allows the sample being pressed to be located in the middle of the induction coil as well as in the same location as the process thermocouple. Next, a graphite spacer is placed in the die, then the sample, another spacer, and finally another piece of graphite rod. After loading the sample into the die and placing the die in the induction coil, the chamber door is closed and a vacuum is applied to the chamber. When an acceptable vacuum has been reached, the desired load is applied to the die, the chamber is backfilled with $\mathrm{Ar}$ and flowed through the chamber, and the induction heating power supply is turned on and heating begins.

In addition to the temperature, the position of the pressing ram is monitored by recording the voltage applied to the hydraulic actuator controlling the hydraulic ram. Pressing takes place in a constant load condition and the pressing ram position is automatically varied to maintain the desired load. The maximum load for this demonstration was $510 \mathrm{~kg}$. As the sample consolidates, the ram will move upward to maintain the desired load, and by monitoring the ram position, the time at which consolidation of the sample is complete can be determined from the time after which the pressing ram stops moving. Determining the time at which the sample is fully consolidated allows for optimization of the pressing procedure by avoiding unnecessary time at elevated temperatures.

\section{RESULTS}

The initial characterization of the RHP was performed using $\mathrm{PbTe}$ that was prepared in our laboratory. Elemental $\mathrm{Pb}$ and Te $(99.999+\%$ purity) were sealed in a quartz vial under $\mathrm{Ar}$ atmosphere and held at $1248 \mathrm{~K}$ for $2 \mathrm{~h}$ and subsequently quenched in water followed by heating to $873 \mathrm{~K}$ and holding for $24 \mathrm{~h}$. The resulting ingot of material was ball milled for

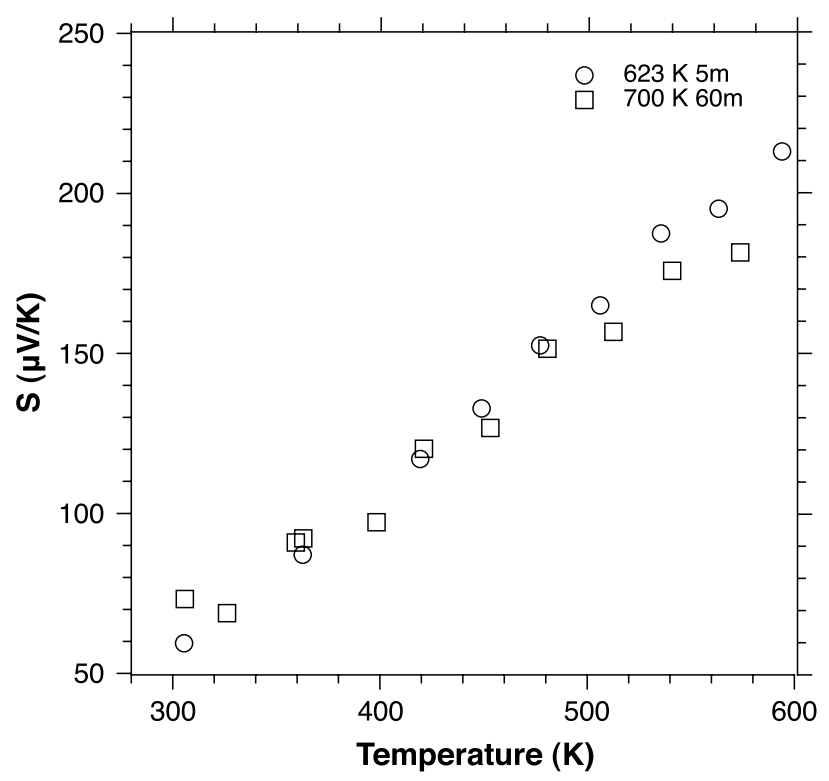

FIG. 3. Comparison of Seebeck coefficient for PbTe:Na (2\%) consolidated at $623 \mathrm{~K}$ for $5 \mathrm{~m}$ and $700 \mathrm{~K}$ for $60 \mathrm{~m}$.
8 h. Material with the composition PbTe: $\mathrm{Na}(2 \%)$ was consolidated using the induction hot press and its thermoelectric properties were measured.

Prior to sample consolidation an initial graphite run was made in which the die arrangement to be used for pressing powder was placed in the hot press without powder included and heated to a pressing temperature of $623 \mathrm{~K}$ while the displacement of the pressing ram was recorded. The initial graphite run takes account of the thermal expansion of the system and allows for full characterization of the pressing profile leading to more accurate determination of the minimum pressing time required to consolidate a sample. Powder of the material to be consolidated was loaded into the die as described above and was heated to $623 \mathrm{~K}$ and held for $10 \mathrm{~min}$. The displacement data from the graphite run were subtracted from the displacement data from the sample run resulting in data representing only the displacement occurring due to the consolidation of the sample. It can be seen in Fig. 2 that the die reaches the pressing temperature after 2 min and during heating the pressing ram is moving, indicating that consolidation is taking place. After a total time of $\sim 7 \mathrm{~min}, 5 \mathrm{~min}$ at $623 \mathrm{~K}$, the displacement has become stable, indicating the minimum time required at this temperature to produce a dense sample. The sample pressed at $623 \mathrm{~K}$ for $10 \mathrm{~min}$ was $98 \%$ of the theoretical density. An additional sample of PbTe was pressed at $623 \mathrm{~K}$ for $5 \mathrm{~min}$ and was found to have a density of $98 \%$ of the theoretical density as well.

Using the results of the PbTe consolidation runs as a guideline for a consolidation process, a sample of $\mathrm{PbTe}: \mathrm{Na}$ (2\%) was consolidated in the RHP at $623 \mathrm{~K}$ for $5 \mathrm{~min}$. The density of the sample was $98 \%$ of the theoretical value. An additional PbTe: $\mathrm{Na}(2 \%)$ sample was pressed at typical consolidation parameters of $700 \mathrm{~K}$ for $60 \mathrm{~m}$, as previously reported with density $>98 \% .{ }^{9}$ The Seebeck coefficient was calculated from the slope of the thermopower versus temperature gradient measurements by Chromel-Nb thermocouples, while

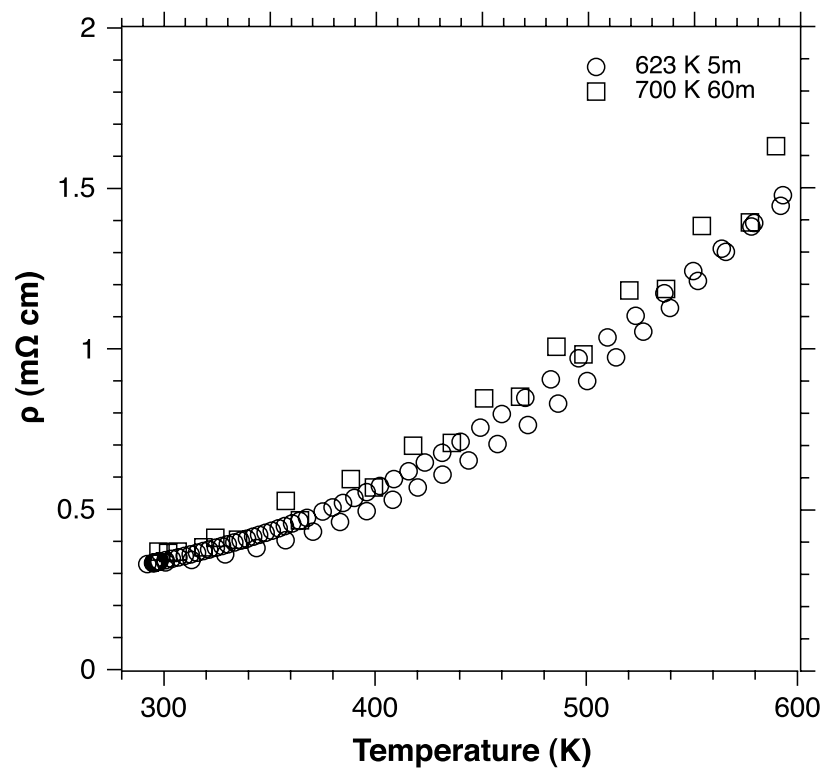

FIG. 4. Comparison of resistivity for $\mathrm{PbTe}: \mathrm{Na}(2 \%)$ consolidated at $623 \mathrm{~K}$ for $5 \mathrm{~m}$ and $700 \mathrm{~K}$ for $60 \mathrm{~m}$. 


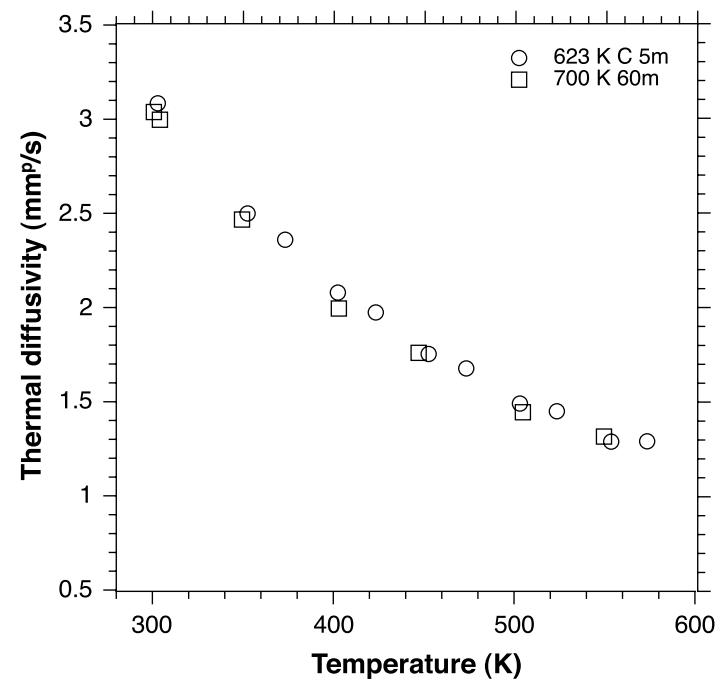

FIG. 5. Comparison of thermal diffusivity for PbTe: $\mathrm{Na}(2 \%)$ consolidated at $623 \mathrm{~K}$ for $5 \mathrm{~m}$ and $700 \mathrm{~K}$ for $60 \mathrm{~m}$.

the resistivity was measured using the Van der Pauw technique under a reversible magnetic field of 2T, and the thermal diffusivity measurement was made by the laser flash method (Netzsch LFA 457). The Seebeck coefficient, resisitivity, and thermal diffusivity were measured for each sample under vacuum, and the heating and cooling data are shown in Figs. 3, 4 and 5. It is demonstrated from the agreement between the presented properties for these two samples that material of equivalent functionality can be produced using the RHP method under pressing conditions that minimize time and temperature required to produce dense thermoelectric material. It can also be noted that the RHP has operated at $1450 \mathrm{~K}$, heating at a rate of $620 \mathrm{~K} / \mathrm{m}$ to produced $\mathrm{SiGe}$ that was $>96 \%$ dense is less than $1 \mathrm{~min}$ of pressing similar to that previously reported. ${ }^{5}$

\section{SUMMARY}

We have designed and fabricated a rapid hot pressing technique based on induction heating that is as fast, if not faster, than SPS for the rapid consolidation of materials without the effects of a dc current. Such a system will be ideal for maintaining small and nanometer scale microstructures. The technique is demonstrated by consolidating dense $\mathrm{PbTe}$ based thermoelectric materials at $623 \mathrm{~K}$ for $5 \mathrm{~min}$ as well as $\mathrm{SiGe}$ based materials by heating to $1450 \mathrm{~K}$ at a rate of $620 \mathrm{~K} / \mathrm{min}$ with similar thermoelectric properties as materials produced using conventional hot pressing for a much longer time. Fabrication and maintenance of this system will cost significantly less than commercial resistance heated or spark plasma sintering presses. The RHP system is an economic and robust system for research, development, and production of materials for a variety of purposes.

\section{ACKNOWLEDGMENTS}

We thank Eric S. Toberer and Espen Flage-Larsen for their contributions to the development of this system. Additionally, we thank Sabah K. Bux for assistance with the SiGe sample and NASA-Jet Propulsion Laboratory and DARPA Nanomaterials program for financial support.

${ }^{1}$ S. Grasso, Y. Sakka, and G. Maizza, Sci. Technol. Adv. Mater. 10, 053001 (2009).

${ }^{2}$ J. Raethel, M. Herrmann, and W. Beckert, J. Eur. Ceram. Soc. 29, 1419 (2009).

${ }^{3}$ Z. Shen, M. Johnsson, Z. Zhao, and M. Nygren, J. Am. Chem. Soc. 85, 1921 (2002)

${ }^{4}$ A. J. Minnich, M. S. Dresselhaus, Z. F. Ren, and G. Chen, Energy Environ. Sci. 2, 466 (2009).

${ }^{5}$ S. Bux, J.-P. Fleurial, R. Blair, P. Gogna, T. Caillat, and R. Kaner, in Materials and Devices for Thermal-to-Electric Energy Conversion, Mater. Res. Symp. Proc., edited by J. Yang, G. Nolas, K. Koumoto, and Y. Grin (Mater. Res. Soc., Warrendale, PA, 2009), Vol. 1166, pp. 53-58.

${ }^{6}$ L. Blackman, P. Dundas, A. Moore, and A. Ubbelohde, Br. J. Appl. Phys. 12, 377 (1961).

${ }^{7}$ M. Beekman, M. Baitinger, H. Borrmann, W. Schnelle, K. Meier, G. S. Nolas, and Y. Grin, J. Am. Chem. Soc. 131, 9642 (2009).

${ }^{8}$ Z. Munir, U. Anselmi-Tamburini, and M. Ohyanagi, J. Mater. Sci. 41, 763 (2006).

${ }^{9}$ Y. Pei, A. LaLonde, S. Iwanaga, and G. J. Snyder, Energy Environ. Sci. (in press). 